\title{
A new method to study ferroelectrics using the remanent Henkel plots
}

\author{
Melvin M. Vopson ${ }^{1 *}$ \\ ${ }^{1}$ Faculty of Science, SEES, University of Portsmouth, Portsmouth PO1 3QL, UK \\ * Correspondence to Dr Vopson: melvin.vopson@port.ac.uk, formerly known as Vopsaroiu
}

\begin{abstract}
Analysis of experimental curves constructed from dc demagnetization and isothermal remanent magnetization known as Henkel and delta M plots, have served for over 53 years as an important tool for characterization of interactions in ferromagnets. In this article we address the question whether the same experimental technique could be applied to the study of ferroelectric systems. The successful measurement of the equivalent dc depolarisation and isothermal remanent polarization curves and the construction of the Henkel and delta P plots for ferroelectrics is reported here. Full measurement protocol is provided together with experimental examples for two ferroelectric ceramic samples. This new measurement technique is an invaluable experimental tool that could be used to further advance our understanding of ferroelectric materials and their applications.
\end{abstract}

\section{Introduction}

The effects of constituent inter-particle interactions within any magnetic medium are not observable in magnetisation cycles such as the major hysteresis loop, but they may be evident in more complex magnetisation processes. The starting point to identify magnetisation curves sensitive to interactions is based on the Wohlfarth relation [1], which links the Isothermal Remanent Magnetisation (IRM), and DC Demagnetisation (DCD) functions. The IRM and DCD curves are measured in a similar way, but their starting points are determined by different initial magnetic states. The IRM curve results from plotting the remanent magnetisation against the applied field, when a sample is initially demagnetised and then the remanent magnetisation measured after each application and removal of an incremental field $\mathrm{H}$, for field values between 0 and saturation. Using a similar method, the DCD curve is produced by measuring the remanent magnetisation as a function of an increasing reverse field applied to a sample at saturation remanent initial state, until its net remanent magnetization reaches reversed saturation remanent state. By noting the DCD dataset as $M_{d c d}(H)$ and the IRM dataset as $M_{\text {irm }}(H)$, it was shown by Wohlfarth [1] that, for an ensemble of non-interacting single-domain uni-axial particles with all atomic moments rotating uniformly, the remanent magnetization functions satisfy the equation:

$M_{d c d}(H)=M_{r}(\infty)-2 \cdot M_{i r p}(H)$

where $M_{r}(\propto)$, also known as $M_{r}(\max )$, is the saturation remanent magnetization. Normalizing relation (1) to $M_{r}(\propto)$ we obtain:

$m_{d c d}(H)=1-2 \cdot m_{\text {irp }}(H)$

where $m_{d c d}(H)$ and $m_{i r m}(H)$ are the remanent magnetization datasets $M_{d c d}(H)$ and $M_{\text {irm }}(H)$ normalized to the $M_{r}(\propto)$. The existence of various inter-particle interactions including demagnetising and magnetizing interactions, the existence of super-paramagnetic particles, multi-domain states, non uni-axial magnetic anisotropy, incoherent magnetization rotation effects and crystallographic defects, would inflict possible deviations from the Wohlfarth equation (2). Henkel was the first who suggested that plotting of $m_{d c d}(H)=f\left(m_{\text {irm }}(H)\right)$ 
dependencies, known as Henkel plots, can demonstrate the above mentioned deviations [2]. Kelly et al. [3] further developed this idea by introducing the following expression:

$$
\delta m(H)=m_{d c d}(H)-\left[1-2 \cdot m_{\text {irm }}(H)\right]
$$

Relation (3) is known as delta $M(\delta m)$ function and its plot versus the applied field is known as $\delta m$ plot, which allows one to perform a quantitative description of deviations from the Wohlfarth equation by plotting $\delta m(H)$ as a function of $H$ values. Both Henkel and $\delta m$ plots are similar methods of measuring the deviations from the Wohlfarth relation, but they show different things. Henkel plots contain no applied field information, so they can be used to compare materials with different coercivity, whereas $\delta m$ plots display interactions as a function of the applied field, which are in fact field dependent quantitative deviations from Wohlfarth relation. For an ideal Wohlfarth medium with no interactions, Henkel plot will present a straight diagonal line and $\delta m$ plot will be zero for all fields. Demagnetising (negative) or magnetizing (positive) interactions within the medium will produce deviations of the Henkel curves from the straight diagonal line and positive or negative $\delta m$ plots [4]. These techniques, and variants of them proposed by Bissell [5], are suitable for both nano-structured and bulk magnetic materials, being highly versatile for experimental studies. Henkel plots [2] have been applied for the description of magnetic interactions in magnetic data storage [6], studies of magnetic rocks [7], studies of superconductors, hard and soft magnets [8], studies of exchange bias systems [9], other studies providing insights on interactions that govern magnetization processes [10-20], or indicate negative interaction processes in systems where no dipolar interactions are present [21, 22].

Given the similarities between the ferromagnetic and ferroelectric materials in that both systems display hysteresis, coercive fields, Curie temperature, and remanent states used in both cases for digital data storage, in this paper it is hypothesized that the equivalent magnetic remanent DCD and IRM curves could be measured for a ferroelectric system and renamed DC Depolarization (DCDP) and Isothermal Remanent Polarization (IRP), respectively. In turn, this would allow extrapolating the application of the Wohlfarth relation to ferroelectric systems and it would facilitate the construction of the Henkel and $\delta m$ plots (renamed $\delta p$ plots) to study the possible dominant interactions within ferroelectric materials. These measurements have been successfully performed here. In the following sections the measurement protocols of the remanent curves, DCDP, IRP, Henkel plots, $\delta p$ plots and polarization switching field distribution curves are presented together with experimental examples on real ferroelectric samples.

\section{Measurement of ferroelectric remanent curves}

Unlike the ferromagnetic systems where remanent states are measured directly in zero applied magnetic field without any difficulty using standard magnetometers, remanent electric polarization states cannot be measured directly. This is because the polarization is determined from integrating the total discharge current during reversal under an applied electric field, so the measurement requires by definition an applied electric field perturbation to create a response. In turn, this results in a change of the remanent polarization state due to the applied field perturbation. The method of extracting remanent curves as a function of the applied voltage (electric field) is by measuring the difference between "switched" and "non-switched" polarization in order to get the remanent states. Figure 1 shows the measurement protocol deployed for the extraction of the polarization remanent curves. This involves the application of a "write" pulse / perturbation of a given amplitude, typically equal to the saturating voltage 
/ E field as determined from a simple polarization hysteresis loop measurement. Two "read" pulses of opposite polarity to the "write" pulse follow the "write" pulse. First pulse will generate the "switched" polarization and the second pulse generates the "non-switched" polarization.

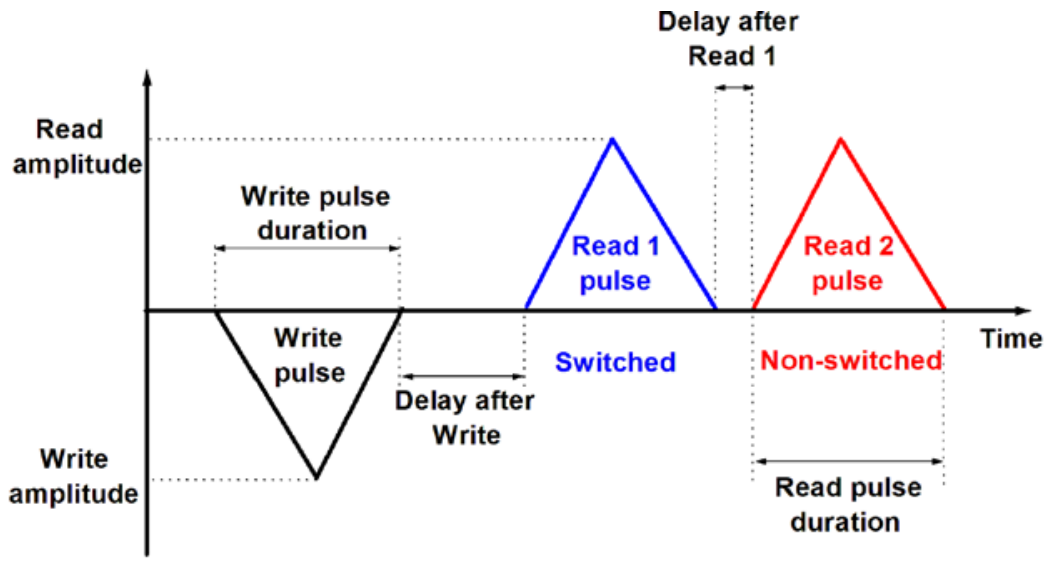

Figure 1. Pulse measurement sequences deployed for the extraction of remanent curves in ferroelectrics. The "write" pulse has the function of setting the initial state of the sample, i.e. a positive or negative saturating pulse will bring the sample to $+\mathrm{P}_{\mathrm{r}}(\max )$ or $-\mathrm{P}_{\mathrm{r}}(\max )$ state, respectively.

Remanent polarization versus voltage is obtained by subtracting "non-switched" data from "switched" data. The resulted data would contain remanent polarization values for both branches of the triangular waveform. However, in our case only the remanent polarization data corresponding to the first branch (rise field) of the triangular waveform is of interest when extracting DCDP and IRP curves. It is important to mention that the "write" and "read" pulses in figure 1 and in our experiments are triangular, but square or trapezoidal electrical pulses can also be applied.

\section{DCDP and IRP experimental data and measurement protocols}

The measurement protocols have been applied to two commercial PZT ceramic samples of different thickness. One sample is a PZT-5H from Fuji. The sample is $20 \mathrm{~mm}$ x $5 \mathrm{~mm}$ x 150 $\mu \mathrm{m}$ thick with $\sim 2 \mu \mathrm{m}$ Ni electrodes. The second sample is a $10 \mathrm{~mm}$ disc shape PZT-5A from Aixact with $500 \mu \mathrm{m}$ thickness and $\sim 5 \mu \mathrm{m}$ Cr electrodes. The polarization hysteresis loops for both samples have been first acquired. The principal parameters extracted from the hysteresis loops are listed in Table 1.

Table 1. Sample parameters extracted from hysteresis polarization loops and switching field distribution values extracted in section 5 .

\begin{tabular}{|c|c|c|c|c|}
\hline Sample & $\begin{array}{c}\mathbf{P}_{\mathbf{r}}(\mathbf{m a x}) \\
\left(\mu \mathrm{C} / \mathrm{cm}^{2}\right)\end{array}$ & $\begin{array}{c}\mathbf{P s}_{\mathrm{S}} \\
\left(\mu \mathrm{C} / \mathrm{cm}^{2}\right)\end{array}$ & $\begin{array}{c}\text { Ec } \\
(\mathrm{kV} / \mathrm{cm})\end{array}$ & $\begin{array}{c}\text { PSFD } \\
(\mathrm{kV} / \mathrm{cm})\end{array}$ \\
\hline PZT 150 $\mu \mathrm{m}$ & 38.96 & 46.56 & 7 & 1.1 \\
\hline PZT $500 \mu \mathrm{m}$ & 35.67 & 42.11 & 10 & 1.13 \\
\hline
\end{tabular}

We begin by allocating the symbols $P_{d c d p}(E)$ and $P_{i r p}(E)$ to the DC Depolarization (DCDP) and Isothermal Remanent Polarization (IRP) datasets, respectively. If $\operatorname{Pr}_{r}(\max )$ is the saturation remanent value, then $p_{d c d p}(E)$ and $p_{i r p}(E)$ are the remanent polarization datasets $P_{d c d p}(E)$ and $P_{\text {irp }}(E)$ normalized to $P_{r}(\max )$. With these notations, Wohlfarth relation for a ferroelectric system of non-interacting electric dipoles and domains is: 
$p_{d c d p}(E)=1-2 \cdot p_{\text {irp }}(E)$

Having these parameters defined, the DCDP and the IRP remanent curves are experimentally produced using the polarization remanent measurement protocol described above. For both samples, the DCDP curve is obtained by applying a triangular positive "write" pulse of rise time $500 \mathrm{~ms}$ and pulse width $2 \mathrm{~s}$. In each case the "write" pulse was a saturating $200 \mathrm{~V}$ or E = $13.3 \mathrm{kV} / \mathrm{cm}$ for the $150 \mu \mathrm{m}$ sample, and $950 \mathrm{~V}$ or $\mathrm{E}=19 \mathrm{kV} / \mathrm{cm}$ for the $500 \mu \mathrm{m}$ sample, respectively. The "write" pulse was followed by a "delay-to-read" of $1 \mathrm{~s}$, and the application of two triangular negative "read" pulses, each of identical amplitude to the "write" pulse, pulse width of $500 \mathrm{~ms}$, and "delay-after-read 1" of $1 \mathrm{~s}$. The sample's initial state is therefore set to maximum positive remanent polarization, $+P_{r}(\max )$, and the DCDP curve represents the remanent depolarization from $+P_{r}(\max )$ to $-P_{r}(\max )$. By subtracting the "non-switching" polarization data from "switching" data a remanent curve function of the applied field, $\operatorname{Pr}_{r}(E)$, is obtained. Since the initial state was $P_{d c d p}(E=0)=+P_{r}(\max )$, the relationship between DCDP, i.e. $P_{d c d p}(E)$ and $P_{r}(E)$ is:

$P_{d c d p}(E)=P_{r}(E)-P_{r}(\max )$

Figure 2.a) shows the remanent curve $\operatorname{Pr}(E)$ for the DCDP protocol applied to the PZT $150 \mu \mathrm{m}$ sample, including the "switching" and "non-switching" polarization curves function of the applied $E$ field. The remanent curves contain data for both branches of the triangular pulses, but the response to the second part (half) of the branch will be excluded when the DCDP $\left(P_{d c d p}(E)\right)$ curve is produced using relation (5) (see figure 3$)$ ).

a)

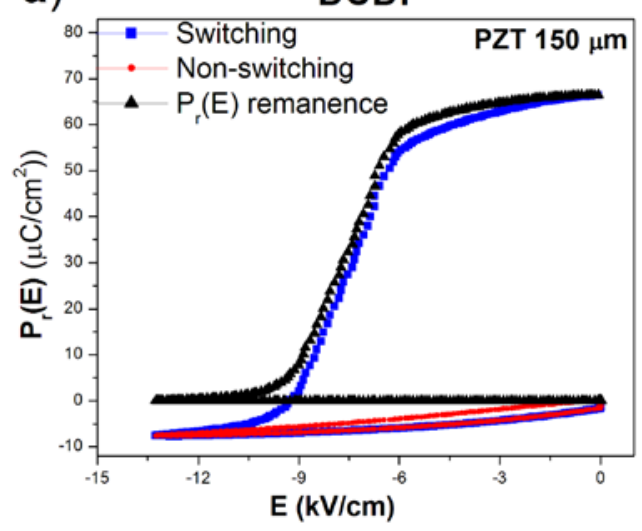

b)

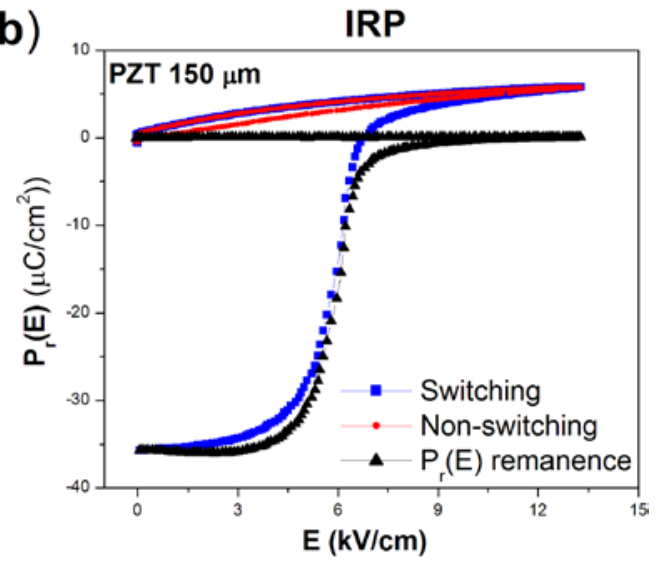

Figure 2. a) Switching, non-switching and remanent polarization curve for the DCDP measurement protocol. b) Switching, non-switching and remanent polarization curve for the IRP measurement protocol. Both data sets refer to a PZT $150 \mu \mathrm{m}$ thick sample.

Unlike DCDP, in the case of IRP measurement, the "write" pulse has zero amplitude and the sample's initial state is zero polarization. This is achieved by heating the sample to a temperature above the Curie temperature, followed by cooling down to room temperature under a decreasing amplitude ac $E$ field applied at $1 \mathrm{~Hz}$ frequency. With the sample depolarized, the remanent measurement protocol is applied with "write" field zero and "read" fields of positive and equal amplitude to the corresponding saturation $E$ field. The "read" field pulses had pulse widths of $500 \mathrm{~ms}$, and "delay-after-read 1" of $1 \mathrm{~s}$ in both cases. Again, subtracting the "nonswitching” polarization data from "switching” data, a remanent curve function of the applied 
field, $\operatorname{Pr}_{r}(E)$, is obtained for the IRP measurement. However, in this case the sample is in depolarized initial state, $P_{\text {irp }}(E=0)=0$, so the relationship between IRP, i.e. $P_{\text {irp }}(E)$ and $P_{r}(E)$ is:

$P_{\text {irp }}(E)=P_{r}(E)+P_{r}(\max )$

Applying (5) and (6) and to the remanent curves in figure 2, excluding the response of the second half of the applied pulse (the horizontal flat lines in each remanent curve in figure 2), and normalizing the datasets to the saturation remanent polarization, $P_{r}(\max )$, we obtain the DCDP and the IRP curves. These normalized remanent curves are shown in figure 3, together with the polarization hysteresis loop. The DCDP is the remanent DC depolarisation curve when a ferroelectric sample is depolarised from an initial remanent state $+P_{r}(\max )$ to a final $-P_{r}(\max )$ state, so the normalized DCDP curve takes values from +1 to -1 and is represented by the function $p_{d c d p}(E)$ in relation (4). The IRP curve is the isothermal remanent polarization curve when a depolarised ferroelectric sample is

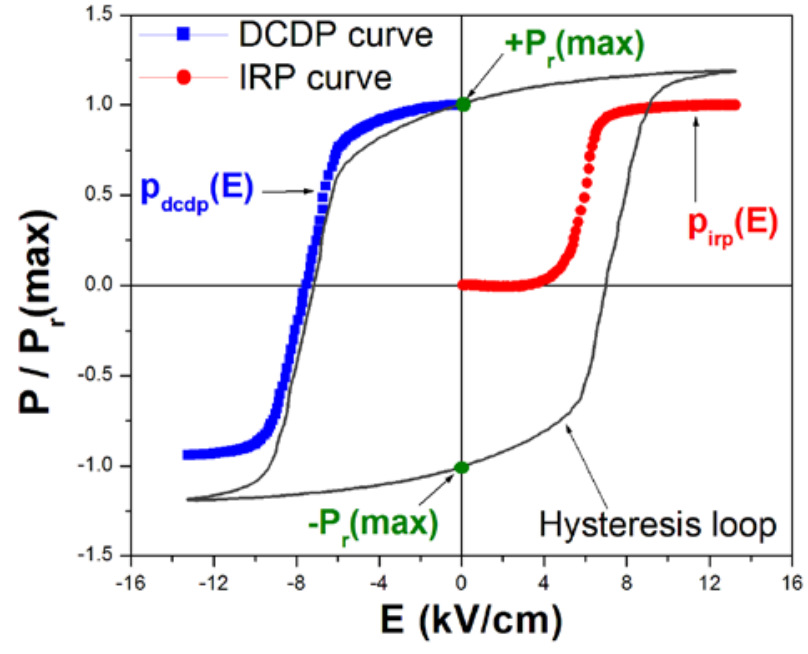

Figure 3. Polarization hysteresis loop, DCDP and IRP remanent curves as a function of the applied electric field for a PZT $150 \mu \mathrm{m}$ sample. polarized from remanent state zero to a final remanent state $+P_{r}(\max )$, so the normalized IRP curve takes values from 0 to 1 and it is represented mathematically by the function $p_{i r p}(E)$ in Wohlfarth relation (4) for a ferroelectric system. It is important to stress that the IRP curve is fundamentally different from the first polarization curve. Besides the data already shown here for the PZT $150 \mu \mathrm{m}$ sample, the experimental procedure to obtain the DCDP and IRP remanent curves has been tested here on a different PZT sample of $500 \mu \mathrm{m}$ thickness. Figures $4 \mathrm{a}$, b show a comparative graph of the combined $p_{d c d p}(E)$ and $p_{i r p}(E)$ data normalized to $P_{r}(\max )$ for both samples.
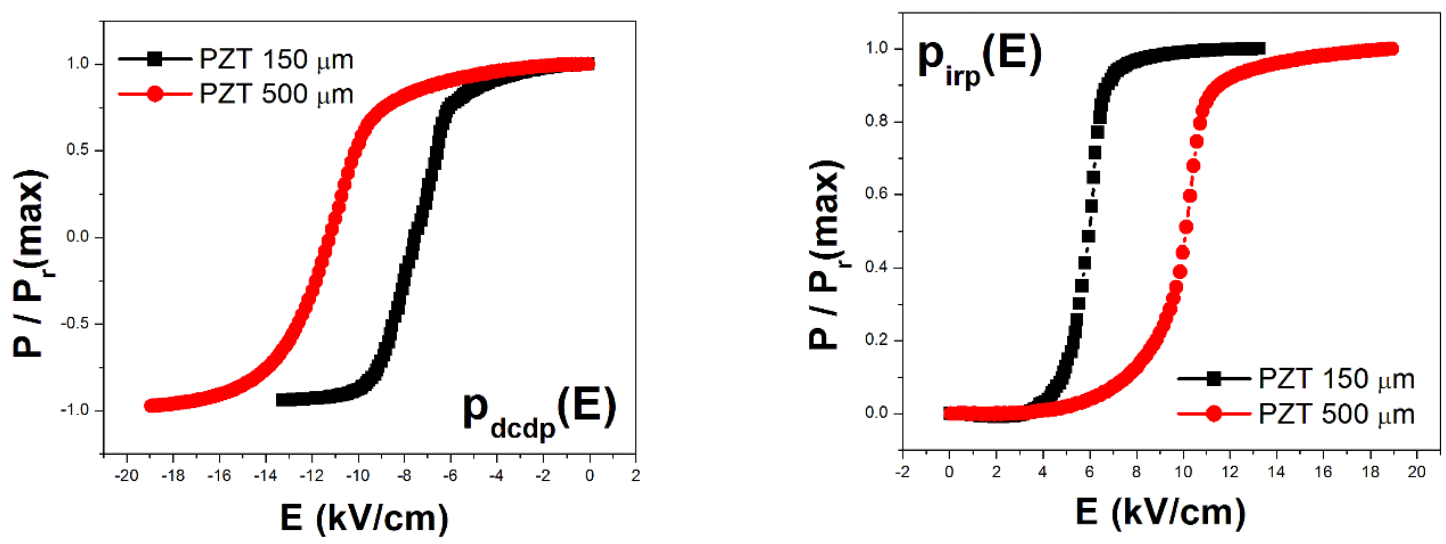

Figure 4. a) $p_{d c d p}(E)$ normalized to $P_{r}(\max )$ graphs corresponding to PZT $150 \mu \mathrm{m}$ and PZT $500 \mu \mathrm{m}$ samples. b) $p_{\text {irp }}(E)$ normalized to $P_{r}(\max )$ graphs corresponding to PZT $150 \mu \mathrm{m}$ and PZT $500 \mu \mathrm{m}$ samples.

Having these remanent curves determined, we can proceed to construct the Henkel and $\delta p$ plots. 


\section{Construction of Henkel and $\delta p$ plots}

The Henkel plot is constructed by plotting the normalized DCDP data versus the normalized IRP data, i.e. $p_{d c d p}$ versus $p_{i r p}$. The Wohlfarth relation (4) for an idealized ferroelectric medium of non-interacting electric dipoles and domains would result in a straight diagonal line Henkel plot (see figure 5). This is the response expected from a perfect ferroelectric single crystal sample, without any impurities, defects, negligible depolarisation charges and noninteracting domains / dipoles. Any imperfections and interactions within the medium would result in a deviation of the Henkel plot from the straight diagonal line. Figure 5 shows the idealized Henkel plot and the experimentally measured Henkel plots for our two PZT ceramic samples. The data indicates, as expected in the case of non-ideal

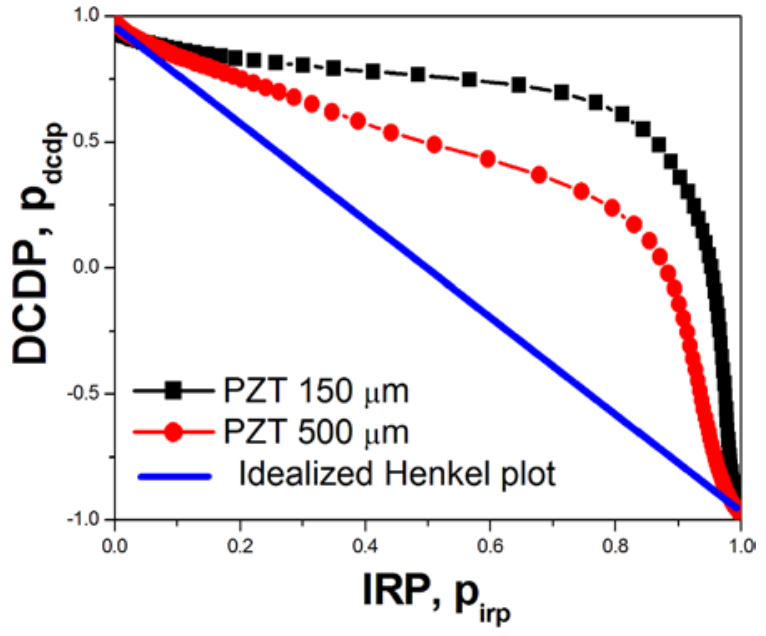

Figure 5. Henkel plots for PZT $150 \mu \mathrm{m}$ and 500 $\mu \mathrm{m}$ PZT samples. samples, positive deviations from the ideal Henkel plot. However, it is interesting to notice that the thinner $150 \mu \mathrm{m}$ ceramic displays more pronounced Henkel plot deviations relative to the $500 \mu \mathrm{m}$ sample, which is a possible indication that interactions in thinner ferroelectrics are more dominant than in bulk, thicker ferroelectrics. The Henkel plots contain no applied field information, but quantitative deviations from Wohlfarth relation can be analysed using $\delta p$ plots that display interactions as a function of the applied $E$ field. From Wohlfarth relation (4), the $\delta p(E)$ function is derived as:

$\delta p(E)=\left(1-2 \cdot p_{i r p}(E)\right)-p_{d c d p}(E)$

From (4) and (7), the following cases are possible:

a) $\delta p(E)=0, p_{d c d p}(E)=1-2 \cdot p_{i r p}(E)$, for a non-interacting ideal ferroelectric;

b) $\delta p(E)<0, p_{d c d p}(E)>1-2 \cdot p_{i r p}(E)$, for a ferroelectric with strong depolarising interactions;

c) $\delta p(E)>0, p_{d c d p}(E)<1-2 \cdot p_{i r p}(E)$, for a ferroelectric with strong polarizing interactions;

Our $\delta p(E)$ data indicates that both samples display $\delta p(E)<0$, suggesting dominant depolarising interactions within the samples. These depolarising interactions can be the result of long range dipole-dipole interactions, as well as the results of a non-

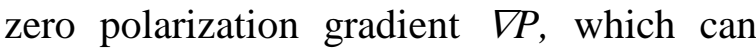
induce a depolarisation field that depends on the interface layer thickness $\left(d_{i n t}\right)$, ferroelectric material thickness $\left(d_{F E}\right)$ and the internal polarization of the ferroelectric $[23,24]$ :

$E_{\text {dep }}=-\frac{d_{\text {int }} D}{d_{F E} \varepsilon_{0} \varepsilon_{\text {int }}}$

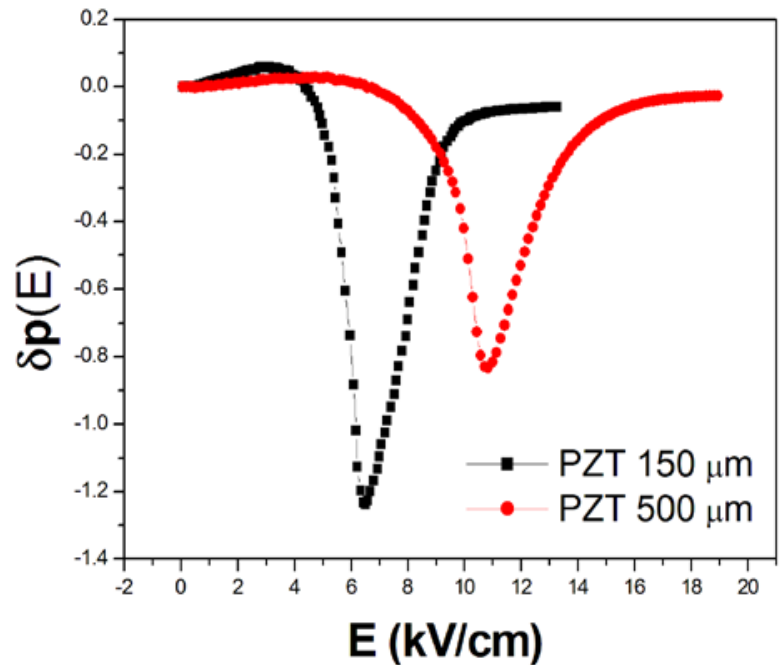

Figure 6. $\delta \mathrm{p}(\mathrm{E})$ plots for PZT $150 \mu \mathrm{m}$ and $500 \mu \mathrm{m}$ PZT samples. 
where: $\varepsilon_{0}=8.85 \times 10^{-12} \mathrm{C} / \mathrm{mV}$; $\varepsilon_{\text {int }}$ is the dielectric constant of the interface layer and the minus sign indicates that the depolarisation field is opposed to the internal polarization of the ferroelectric. Equation (8) indicates that depolarising interactions can be significant for thinner ferroelectrics due to the thickness $d_{F E}$ term in the denominator. This is exactly what we observed in our measurements, where $\delta p(E)$ plot for the $150 \mu \mathrm{m}$ ceramic sample shows more pronounced demagnetising interactions than that of the $500 \mu \mathrm{m}$ sample. While for large thickness / bulk ferroelectrics the depolarising field can be negligible, the variability of the strength in these small interactions can be detected via the $\delta p(E)$ plots, as shown in figure 6.

\section{Polarization Switching Field Distribution (PSFD)}

Although both magnetic IRM and DCD curves, or polar IRP and DCDP curves, provide no useful parameters that can be extracted directly from these measurements, it has been proven that they are very useful for providing a measure of intrinsic interactions via the Henkel plots and delta plots. In addition, it has been shown that another useful parameter derived from these remanent measurements is the switching field distribution [25], which is closely related to the coercive field of a given ferroic material, but it provides a detailed picture of the distribution of possible reversal fields. An ideal medium with square hysteresis loop would display a single value switching field distribution equal to the coercive field and shaped as a Dirac delta function, while a real medium displays a distribution of switching fields due to variations such as particle volumes and inter-particles interactions, with the distribution of the switching fields centred on the coercive field value as extracted from the hysteresis measurements. In the case of magnetic systems, Chantrell and O'Grady proposed and demonstrated that differentiation of the Isothermal Remanent Magnetization curve in respect with the applied field provides a detailed measure of the Magnetic Switching Field Distribution (MSFD) [25]. The authors proposed a quantitative measure of the MSFD, which can be directly measured from this plot as full width at half maximum of the MSFD curve. Here we applied the same method to extract the polarization switching field distribution (PSFD) for our two samples as:

$$
P S F D=\frac{\partial p_{i r p}(E)}{\partial E}
$$

This was achieved by directly differentiating the IRP datasets, i.e. the function $p_{\text {irp }}(E)$ in respect with the applied electric fields. It is important to mention that differentiation works only if a sufficient number of data points are contained in the original IRP curve. For measurements where the number of points is not large enough, a simple interpolation of the dataset with an increased number of points can easily solve this problem without losing any physical validity.

Figure 7 shows the polarization switching field distributions (PSFD) for PZT $150 \mu \mathrm{m}$ and $500 \mu \mathrm{m}$ ceramic samples. The coercive field of PZT $150 \mu \mathrm{m}$ is $7 \mathrm{kV} / \mathrm{cm}$ and 10 $\mathrm{kV} / \mathrm{cm}$ for the PZT $500 \mu \mathrm{m}$, respectively (see Table 1). The PSFD curves in figure 7 show a peak at exactly the value corresponding to

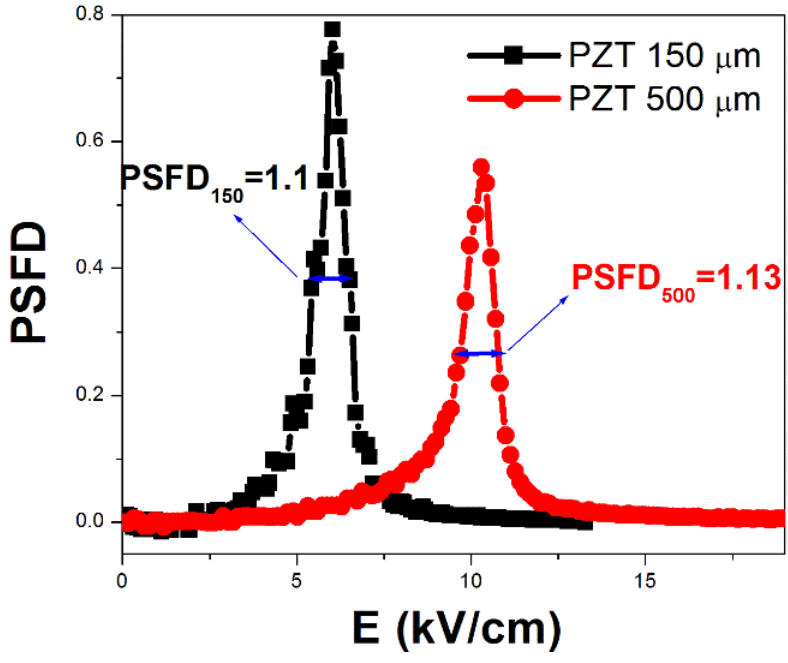

Figure 7. PSFD plots for PZT $150 \mu \mathrm{m}$ and $500 \mu \mathrm{m}$ PZT samples. 
the coercive field of each sample, and a range of switching fields distributed around the main coercive field value. The values of the full width at half maximum of the PSFD curves are 1.1 $\mathrm{kV} / \mathrm{cm}$ for PZT $150 \mu \mathrm{m}$ and $1.3 \mathrm{kV} / \mathrm{cm}$ for PZT $500 \mu \mathrm{m}$, respectively. This indicates that, although the thinner PZT $150 \mu \mathrm{m}$ sample shows stronger interactions, it has a narrower PSDF profile than that of the PZT $500 \mu \mathrm{m}$. The PSFD profiles are very important for ferroelectric random access memories (FRAM) applications [26-28], where a sharp well-defined switching field is highly desirable.

\section{Conclusions}

Experimental characterization of polar dielectrics is essential to advance our understanding of their polarization dynamics and to further improve their commercial applicability [29]. The main experimental measurement tools involving polarization hysteresis measurements, dielectric constant measurements, piezo-displacement measurements and various microscopy or structural measurements under various external fields, stress, time and temperature [30,31] conditions are indeed very valuable. However, complex intrinsic interactions are not visible in these standard measurements and more specialized experimental tools are required. For over half a century, interactions in magnetic systems have been successfully characterized using experimental curves constructed from dc demagnetisation and isothermal remanent magnetization known as Henkel and delta M plots, derived from the Wohlfarth relation (1). In this article it has been shown, for the first time, that the same experimental techniques could be applied to the study of ferroelectric systems, and the successful measurement of the dc depolarisation and isothermal remanent polarization curves, as well as the construction of the Henkel plots, delta P plots and polarization switching field distribution for ferroelectrics, are reported here. These measurements have been tested on two PZT ceramic samples of different thickness and composition. While these measurements are extremely beneficial as an additional experimental tool, further work is required to understand the true value of these measurements and the limits of their applicability. For example in the case of magnetic systems, negative interactions are associated with the dipole-dipole long-range interactions and other demagnetising interactions for which an electrical equivalent is easily identified. However, positive interactions in magnetic systems are associated with short range Heisenberg exchange coupling interactions, for which there is no polar equivalent. Nevertheless, the proposed measurements are expected to generate significant impact as they could be invaluable tools for future studies of depolarising effects [32,33], relaxors [34], defects in ferroelectrics [35,36], ferroelectric hetero-structures [37], geometrical, size, topological and thickness effects in ferroelectrics [38-41], surface and interfaces effects [42,43], dead layer effects [44] and even other polar ordered systems such as anti-ferroelectrics [45,46] and multiferroics [47]. Extensions of these studies to emulate other proposed methods used to extract information about the interactions in magnetic systems [48,49], or the measurement of Interaction Field Factor (IFF) [50], could also be adopted in future ferroelectrics studies.

\section{References}

[1] E. P. Wohlfarth, Relations between different modes of acquisition of the remanent magnetization of ferromagnetic particles, J. Appl. Phys. 29 (1958) 595-596.

[2] O. Henkel, Physica Status Solidi 7 (1964) 919.

[3] P. E. Kelly, K. O'Grady, P. I. Mayo, R. W. Chantrell, Switching mechanisms in Cobalt-phosphorus thin films, IEEE Trans. Magn. 25 (1989) 3881-3883.

[4] M. Vopsaroiu, P.R. Bissell, Interaction effects on the anisotropy field in sputtered Co-Cr-Ta thin films and metal particle tapes, Journal of Physics D: Applied Physics, 35, pp. 1296-1300 (2002) 
[5] P. R. Bissell, J. Magn. Society of Japan, 18, Supp. S1, 261 (1994).

[6] S. Thamm, J. Hesse, J. Magn. Magn. Mater. 154 (1996) 254.

[7] P. Hejda, E. Petrovsky, T. Zelinka, IEEE Trans. Magn. 30 (1994) 896.

[8] V. Basso, G. Bertotti, IEEE Trans. Magn. 30 (1994) 64.

[9] A. Harres, R. Cichelero, L. G. Pereira, J. E. Schmidt, J. Geshev, Remanence plots technique extended to exchange bias systems, J. Appl. Phys. 114 (2013) 043902 (1) - (5).

[10] V. Basso, M. Lo Bue, G. Bertotti, J. Appl. Phys.75 (1994) 5677.

[11] F. Vajda, E. Della Torre, R. D. McMichael, J. Appl. Phys.75 (1994) 5689.

[12] C. J. Buehler, I. D. Mayergoyz, J. Appl. Phys. 79 (1996) 5746.

[13] K.-H. Müller, D. Eckert, A. Handstein, P. Nothnanagel, Deviations from Wohlfarth's remanence relationship in NdFeB magnets, J. Magn. Magn. Mater. 104 - 107(1992)1173-1174.

[14] B.Z. Cui, M. J. O'Shea, Hard magnetic properties of rapidly annealed NdFeB/Co films and inter-grain interactions, J. Magn. Magn. Mater. 279 (2004) 27-35.

[15] J. Geshev, M. Mikhov, Remanence curves for a disordered system of three-and four-axial fine particles. Henkel-type plots, J. Magn. Magn. Mater.104-107 (1992) 1569 - 1570.

[16] J. Garcia-Otero, M. Porto, J. Rivas, Henkel plots of single-domain ferromagnetic particles, J. Appl. Phys. 87 (2000) 7376-7381.

[17] M. E. Mata Zamora, H. Montiel, G. Alvarez, J. F. Barrona, H. Arriola, J. M. Saniger, R. Valenzuela, Remanence of the interparticle interactions and its influence on the microwave absorption in Co-ferrite, J. Magn. Magn. Mater. 320 (2008) e139-e141.

[18] A. Xia, H. Ge, C. Chang, W. Chang, B. Han, Magnetic and crystalline micro-structures of FeB/FePt-type nanocomposite ribbons with high permanent properties, J. Magn. Magn. Mater. 305 (2006) 336-341.

[19] H. Zhang, C. Rong, X. Du, J. Zhang, S. Zhang, B. Shen, Investigation on intergrain exchange coupling of nanocrystalline permanent magnets by Henkel plot, Appl. Phys. Lett. 82 (2003) 4098-4100.

[20] V. Neu, R. Biele, A. Singh, L. Schultz, Modeling of intergrain exchange coupling for quantitative predictions of $\delta$ M plots, IEEE Trans. Magn. 46 (2010) 2331-2333.

[21] J.A. De Toro, M. Vasilakaki, S.S. Lee, M.S. Andersson, P.S. Normile, N. Yaacoub, P. Murray, E.H.

Sanchez, P. Muniz, D. Peddis, R. Mathieu, K. Liu, J. Geshev, K.N. Trohidou, J. Nogues, Remanence Plots as a Probe of Spin Disorder in Magnetic Nanoparticles, Chem. Mater., 29 (19), pp 8258-8268, (2017).

[22] R. Cichelero, A. Harres, K.D. Sossmeier, J.E. Schmidt, J. Geshev, Magnetic interactions in exchangecoupled yet unbiased IrMn/NiCu bilayers, J. Phys.: Condens. Matter 25, 426001, (2013).

[23] X.J. Lou, J. Phys: Condes. Matter 21 (2009) 012307

[24] J.M. Benedetto, R.A. Moore, F.B. McLean, J. Appl. Phys. 75 (1) 460-466 (1994)

[25] R.W. Chantrell, K. O’Grady, J. Phys. D: Appl. Phys. 25 (1992), pp. 1-23

[26] C.P. Yeh, et al., Fabrication and investigation of three-dimensional ferroelectric capacitors for the application of FeRAM, AIP ADVANCES 6, 035128 (2016)

[27] H. Ishiwara, M. Okuyama, Y. Arimoto, Ferroelectric Random Access Memories: Fundamentals and Applications, Springer Science, 16 Apr. 2004

[28] H. Ishiwara, Ferroelectric Random Access Memories, J. Nanosci. Nanotechnol. 12, 7619-7627, (2012)

[29] J.F. Scott, Application of modern ferroelectrics, Science 315, 954-959 (2007)

[30] M. Vopsaroiu, J. Blackburn, M. G. Cain, P. M. Weaver, Phys. Rev. B, vol. 82, 024109 (2010)

[31] M. Vopsaroiu, P.M. Weaver, M.G. Cain, M.J. Reece, K.B. Chong, IEEE Transactions on Ultrasonics, Ferroelectrics, and Frequency Control, vol. 58, no. 9, 1867-1873 (2011)

[32] P. Wurfel, I. P. Batra, Depolarization-Field-Induced Instability in Thin Ferroelectric Films-Experiment and Theory, Phys. Rev. B 8, 5126 (1973)

[33] M. Dawber, P. Chandra, P. B. Littlewood, J. F. Scott, Depolarization corrections to the coercive field in thin-film ferroelectrics, Journal of Physics: Condensed Matter, Volume 15, Number 24 (2003)

[34] Zdravko Kutnjak, Robert Blinc, and Y. Ishibashi, Electric field induced critical points and polarization rotations in relaxor ferroelectrics, Phys. Rev. B 76, 104102 (2007)

[35] C. Elissalde, J. Ravez, Ferroelectric ceramics: defects and dielectric relaxations, J. Mater. Chem., 11, issue 8, 1957-1967 (2001)

[36] Peng Gao, Christopher T. Nelson, Jacob R. Jokisaari, Seung-Hyub Baek, Chung Wung Bark, Yi Zhang, Enge Wang, Darrell G. Schlom, Chang-Beom Eom, Xiaoqing Pan, Revealing the role of defects in ferroelectric switching with atomic resolution, Nature Communications vol. 2, 591 (2011)

[37] M. Tyunina, Size effects and dielectric behaviour in ferroelectric heterostructures, Journal of Physics: Condensed Matter, Volume 18, Number 24 (2006)

[38] Shaoping Li, Jeffery A. Eastman, James M. Vetrone, Christopher M. Foster, Robert E. Newnham, L. Eric Cross, Dimension and Size Effects in Ferroelectrics, Jpn. J. Appl. Phys., Vol. 36, Part 1, No. 8, 5169 (1997) [39] M. Alexe, C. Harnagea, D. Hesse, U. Gösele, Polarization imprint and size effects in mesoscopic ferroelectric structures, Appl. Phys. Lett. 79, 242 (2001) 
[40] W. L. Zhong, Y. G. Wang, P. L. Zhang, B. D. Qu, Phenomenological study of the size effect on phase transitions in ferroelectric particles, Phys. Rev. B 50, 698 (1994)

[41] C. H. Ahn, K. M. Rabe,J.-M. Triscone, Ferroelectricity at the Nanoscale: Local Polarization in Oxide Thin Films and Heterostructures, Science, Vol. 303, Issue 5657, pp. 488-491 (2004)

[42] M. H. Frey , Z. Xu , P. Han, D. A. Payne, The role of interfaces on an apparent grain size effect on the dielectric properties for ferroelectric barium titanate ceramics, Ferroelectrics Vol. 206, Iss. 1, 337-353 (1998) [43] Y. G. Wang, W. L. Zhong, and P. L. Zhang, Surface and size effects on ferroelectric films with domain structures, Phys. Rev. B 51, 5311 (1995)

[44] Y. Wang, M. K. Niranjan, K. Janicka, J. P. Velev, M. Ye. Zhuravlev, S. S. Jaswal, and E. Y. Tsymbal, Ferroelectric dead layer driven by a polar interface, Phys. Rev. B 82, 094114 (2010)

[45] M. Vopson, G. Caruntu, X. Tan, Polarization reversal and memory effect in anti-ferroelectric materials, Scripta Materialia, vol. 128, 61-64, (2017).

[46] M. Vopson, X. Tan, Non-equilibrium polarization dynamics in anti-ferroelectrics, Phys Rev. B, 96, 014104 (2017).

[47] M. M. Vopson, Y. K. Fetisov, G. Caruntu, G. Srinivasan, Measurement Techniques of the MagnetoElectric Coupling in Multiferroics, Materials 2017, 10(8), 963

[48] S. Thamm, J. Hesse, The remanence of a Stoner-Wohlfarth particle ensemble as a function of demagnetisation process, Journal of Magnetism and Magnetic Materials 184(2):245-255 (1998)

[49] V.Masheva, J. Geshev, M. Mikhov, Fourier analysis of hysteresis loops and initial magnetization curves: application to the singular-point-detection method, Journal of Magnetism and Magnetic Materials 137(3):350357 (1994)

[50] Xiao-dong Che, H.N. Bertram, Phenomenology of $\delta \mathrm{M}$ curves and magnetic interactions, Journal of Magnetism and Magnetic Materials 116(1-2):121-127 (1992)

\section{Acknowledgements}

The author would like to acknowledge the valuable discussions he had with Prof. Kevin O'Grady, from the University of York on various aspects of this article, as well as the financial research support from EPSRC grant EP/R028656/1 and Diamond Light Source grant EE18495, on closely related projects that stimulated this work. 